\title{
On the Join Operation of Graphs Obtained by Monogenic Semigroups
}

\author{
YAŞAR NACAROĞLU iD \\ Department of Mathematics, Faculty of Science and Letters, Kahramanmaras Sutcu Imam University, 46100, Kahramanmaras, \\ Turkey.
}

Received: 22-06-2020 • Accepted: 23-03-2021

\begin{abstract}
For each commutative ring $R$ we associate a simple graph $\Gamma(R)$. This relationship presents a link between algebra and graph theory. Our main scope in this study is to extend this study over the special algebraic graphs to join graph operations. In this paper, we will give some graph parameters for the join of monogenic semigroup graphs.
\end{abstract}

2010 AMS Classification: $05 \mathrm{C} 10,05 \mathrm{C} 12,06 \mathrm{~A} 07,15 \mathrm{~A} 18,15 \mathrm{~A} 36$

Keywords: Join operation, monogenic semigroup graphs, graph parameters.

\section{INTRODUCTION}

In [6], Beck put forward the opinion of the zero divisor graph. This concept has been studied in various forms to the present day. Similarly, this concept was defined in semigroups and many studies were done $[3-5,8,9]$.

In a recently study Das et.al [7], the graph $\Gamma\left(S_{M}\right)$ defined. They considered the monogenic semigroup (with zero) $S_{M}=\left\{0, x, x^{2}, \ldots, x^{p}\right\}$. In here, $V\left(\Gamma\left(S_{M}\right)\right)=\left\{x, x^{2}, \ldots, x^{p}\right\}$ and the vertices $x^{i}$ and $x^{j}$ are adjacent with the rule $x^{i} \cdot x^{j}=0$ if and only if $i+j \geq p+1,(1 \leq i, j \leq p)$. The other words $x^{i} \sim x^{j}$ iff $i+j \geq p+1,(1 \leq i, j \leq p)$.

In previous studies, The Cartesian product, the lexicographic product, the strong product, the disjunctive product and the corona product of monogenic semigroup graphs were defined and some properties were given in $[1,2,7,13,14]$.

In this paper, by considering join operation of monogenic semigroup graphs we will give some results for some graph parameters such as the diameter, radius, girth etc.

\section{Main Results}

The join of two graphs $G_{1}$ and $G_{2}$ is denoted by $G_{1}+G_{2}$. It has the following vertex-set and edge set:

$$
V\left(G_{1}+G_{2}\right)=V\left(G_{1}\right) \cup V\left(G_{2}\right) \text { and } E\left(G_{1}+G_{2}\right)=E\left(G_{1}\right) \cup E\left(G_{2}\right) \cup\left\{i j: i \in V\left(G_{1}\right) \text { and } j \in V\left(G_{2}\right)\right\} \text {. }
$$

In here we replace $G_{1}$ by $\Gamma\left(S_{M}^{1}\right)$ and $G_{2}$ by $\Gamma\left(S_{M}^{2}\right)$, where $S_{M}^{1}=\left\{x, x^{2}, \ldots, x^{p}\right\}$ with 0 and $S_{M}^{2}=\left\{y, y^{2}, \ldots, y^{s}\right\}$ with 0 . We have rules for monogenic semigroup graphs as follows:

Email address: yasarnacaroglu@ksu.edu.tr (Y. Nacaroğlu) 
$\Gamma\left(S_{M}^{1}\right)+\Gamma\left(S_{M}^{1}\right)$ has vertex set $V\left(\Gamma\left(S_{M}^{1}\right)+\Gamma\left(S_{M}^{2}\right)\right)=V\left(\Gamma\left(S_{M}^{1}\right)\right) \cup V\left(\Gamma\left(S_{M}^{2}\right)\right)$ and let us take any two vertices of $\Gamma\left(S_{M}^{1}\right)+\Gamma\left(S_{M}^{2}\right)$ are adjacent if and only if

$$
\left\{\begin{array}{c}
x^{i} x^{j}=0 \Leftrightarrow i+j \geq p+1, \quad \text { where } x^{i}, x^{j} \in V\left(\Gamma\left(S_{M}^{1}\right)\right. \\
y^{i} y^{j}=0 \Leftrightarrow i+j \geq s+1, \quad \text { or where } y^{i}, y^{j} \in V\left(\Gamma\left(S_{M}^{2}\right)\right. \\
x^{i} y^{j}=0 \Leftrightarrow i+j \geq 2, \quad \text { where } x^{i} \in V\left(\Gamma ( S _ { M } ^ { 1 } ) \text { and } y ^ { j } \in V \left(\Gamma\left(S_{M}^{2}\right)\right.\right.
\end{array}\right.
$$

In the following parts of the study, we will use the $\Gamma_{1}$ and $\Gamma_{2}$ notation instead of $\Gamma\left(S_{M}^{1}\right)$ and $\Gamma\left(S_{M}^{2}\right)$ for convenience, respectively. Now, we give some properties of the graph $\Gamma_{1}+\Gamma_{2}$.

For two vertices $i$ and $j$ of $V(G)$ we define their distance $d_{G}(i, j)$ as the length of a shortest path connecting $i$ and $j$ in $G$. The diameter of $G$ is defined as $\operatorname{diam}(G)=\max \{d(i, j): i, j \in V(G)\}[10]$.

Theorem 2.1. Let $S_{M}^{1}=\left\{0, x, x^{2}, \ldots, x^{p}\right\}$ and $S_{M}^{2}=\left\{0, y, y^{2}, \ldots, y^{s}\right\}$ be two monogenic semigroups. Then we have

$$
\operatorname{diam}\left(\Gamma_{1}+\Gamma_{2}\right)=2 \text {. }
$$

Proof. We know that $x^{i} \sim x^{p}$ for all $1 \leq i \leq p-1$ and $y^{j} \sim y^{s}$ for all $1 \leq j \leq s-1$. Also from definition of join operation we have $x^{i} \sim y^{j}$ for all $1 \leq i \leq p$ and $1 \leq j \leq s$. Then we get $\operatorname{diam}\left(\Gamma_{1}+\Gamma_{2}\right)=2$.

If $i \in V(G)$ is a vertex of $G$, its eccentricity $e(i)$ is defined as $e(i)=\max \{d(i, j): j \in V(G)\}$. The radius of $G$ is defined as $\operatorname{radius}(G)=\operatorname{rad}(G)=\min \{e(i): i \in V(G)\}[10]$.

Theorem 2.2. Let $S_{M}^{1}=\left\{0, x, x^{2}, \ldots, x^{p}\right\}$ and $S_{M}^{2}=\left\{0, y, y^{2}, \ldots, y^{s}\right\}$ be two monogenic semigroups. Then we have

$$
\operatorname{rad}\left(\Gamma_{1}+\Gamma_{2}\right)=1 \text {. }
$$

Proof. We easily see that the vertex $x^{p}$ of $\Gamma_{1}$ is adjacent to all other vertices of $\Gamma_{1}$. Moreover, by the definition of join operation this vertex is adjacent to all vertices of $\Gamma_{2}$. Finally, we get $\operatorname{rad}\left(\Gamma_{1}+\Gamma_{2}\right)=1$

The girth of a graph $G$ is the length of a shortest cycle in $G$. If the graph $G$ does not contain any cycle, then the girth is taken as infinite [10].

Theorem 2.3. Let $S_{M}^{1}=\left\{0, x,{ }^{2}, \ldots, x^{p}\right\}$ and $S_{M}^{2}=\left\{0, y, y^{2}, \ldots, y^{s}\right\}$ be two monogenic semigroups. Then the girth of the graph $\Gamma_{1}+\Gamma_{2}$ is 3 .

Proof. By the definition of join operation, we have $x^{i} \sim x^{p}$ for all $1 \leq i \leq p-1$ and $y^{j} \sim y^{s}$ for all $1 \leq j \leq s-1$. Also we have $x^{i} \sim y^{j}$ for all $1 \leq i \leq p$ and $1 \leq j \leq s$. That is $x^{i} \sim x^{p} \sim y^{j} \sim x^{i}$ for every $i=1,2, \ldots, p$ and $j=1,2, \ldots, s$. Then the girth of the graph $\Gamma_{1}+\Gamma_{2}$ is 3 .

The number of edges incident at $i$ in $G$ is called the degree of the vertex $i$ in $G$ and is denoted by $d_{G}(i)$. Among all degrees, the maximum $\Delta(G)$ (or the minimum $\delta(G)$ ) degrees of $G$ is the number of the largest (or smallest) degree in $G[10]$.

Theorem 2.4. Let $S_{M}^{1}=\left\{0, x, x^{2}, \ldots, x^{p}\right\}$ and $S_{M}^{2}=\left\{0, y, y^{2}, \ldots, y^{s}\right\}$ be two monogenic semigroups. Then

$$
\Delta\left(\Gamma_{1}+\Gamma_{2}\right)=p+s-1
$$

Proof. Let us take the vertex $x^{p}$ of $\Gamma_{1}$. It is clear that $x^{p} \sim x^{i}$ for any $1 \leq i \leq p-1$. By the definition join operation we have $x^{p} \sim y^{j}$ for any $1 \leq j \leq s$. So we get $\operatorname{deg}\left(x^{p}\right)=p-1+s$. Now let us take the vertex $y^{s}$ of $\Gamma_{2}$. By similar steps we get $\operatorname{deg}\left(y^{s}\right)=s-1+p$. Thus, we have $\Delta\left(\Gamma_{1}+\Gamma_{2}\right)=p+s-1$.

Theorem 2.5. Let $S_{M}^{1}=\left\{0, x, x^{2}, \ldots, x^{p}\right\}$ and $S_{M}^{2}=\left\{0, y, y^{2}, \ldots, y^{s}\right\}$ be two monogenic semigroups. Then

$$
\delta\left(\Gamma_{1}+\Gamma_{2}\right)=\min \{p+1, s+1\} .
$$

Proof. Among the vertices of $V\left(\Gamma_{1}+\Gamma_{2}\right)$, let us take vertices $x \in V\left(\Gamma_{1}\right)$ and $y \in V\left(\Gamma_{2}\right)$. The vertex $x$ is adjacent to the vertex $x^{p}$ in $\Gamma_{1}$ and all vertices in $\Gamma_{2}$. Also the vertex $y$ is adjacent to the vertex $y^{s}$ in $\Gamma_{2}$ and all vertices in $\Gamma_{1}$. That is, $\operatorname{deg}(x)=s+1$ and $\operatorname{deg}(y)=p+1$. Thus we get $\delta\left(\Gamma_{1}+\Gamma_{2}\right)=\min \{p+1, s+1\}$. So as desired. 
The degree sequence of a graph is the non-increasing sequence of the degrees of the vertices. The irregularity index is equal to the number of distinct elements in the degree sequence. The degree sequence and irregularity index of the graph $G$ are denoted by $D S(G)$ and $t(G)$, respectively [13].

Theorem 2.6 ( [1]). Let $S_{M}$ be a monogenic semigroup. Then

$$
D S\left(\Gamma\left(S_{M}\right)\right)=\left\{1,2,3, \ldots,\left\lfloor\frac{p}{2}\right\rfloor-1,\left\lfloor\frac{p}{2}\right\rfloor,\left\lfloor\frac{p}{2}\right\rfloor,\left\lfloor\frac{p}{2}\right\rfloor+1,\left\lfloor\frac{p}{2}\right\rfloor+2, \ldots, p-2, p-1\right\}
$$

and $t\left(\Gamma\left(S_{M}\right)\right)=p-1$, respectively.

Theorem 2.7. Let $S_{M}^{1}=\left\{0, x, x^{2}, \ldots, x^{p}\right\}$ and $S_{M}^{2}=0, y, y^{2}, \ldots, y^{s}$ be two monogenic semigroups with $p \geq s$. Then the degree sequence of $G=\Gamma_{1}+\Gamma_{2}$ is given by

$$
\begin{aligned}
D S(G)= & \left\{1+s, 2+s, 3+s, \ldots,\left\lfloor\frac{p}{2}\right\rfloor-1+s,\left\lfloor\frac{p}{2}\right\rfloor+s,\left\lfloor\frac{p}{2}\right\rfloor+s,\left\lfloor\frac{p}{2}\right\rfloor+1+s,\left\lfloor\frac{p}{2}\right\rfloor+2+s, \ldots, p-2+s, p-1+s, 1+p,\right. \\
& \left.2+p, 3+p, \ldots,\left\lfloor\frac{s}{2}\right\rfloor-1+p,\left\lfloor\frac{s}{2}\right\rfloor+p,\left\lfloor\frac{s}{2}\right\rfloor+p,\left\lfloor\frac{s}{2}\right\rfloor+1+p,\left\lfloor\frac{s}{2}\right\rfloor+2+p, \ldots, s-2+p, s-1+p\right\}
\end{aligned}
$$

and $t\left(\Gamma_{1}+\Gamma_{2}\right)=p-1$, respectively.

Proof. By the definition of join product and Theorem 2.6, we get the degree sequence of $\Gamma_{1}+\Gamma_{2}$ as follows:

$$
\begin{aligned}
D S(G)= & \left\{1+s, 2+s, 3+s, \ldots,\left\lfloor\frac{p}{2}\right\rfloor-1+s,\left\lfloor\frac{p}{2}\right\rfloor+s,\left\lfloor\frac{p}{2}\right\rfloor+s,\left\lfloor\frac{p}{2}\right\rfloor+1+s,\left\lfloor\frac{p}{2}\right\rfloor+2+s, \ldots, p-2+s, p-1+s, 1+p,\right. \\
& \left.2+p, 3+p, \ldots,\left\lfloor\frac{s}{2}\right\rfloor-1+p,\left\lfloor\frac{s}{2}\right\rfloor+p,\left\lfloor\frac{s}{2}\right\rfloor+p,\left\lfloor\frac{s}{2}\right\rfloor+1+p,\left\lfloor\frac{s}{2}\right\rfloor+2+p, \ldots, s-2+p, s-1+p\right\}
\end{aligned}
$$

Moreover, since $p \geq s$, the irregularity index of the graph $\Gamma_{1}+\Gamma_{2}$ is $t\left(\Gamma_{1}+\Gamma_{2}\right)=(p-1+s)-(1+s)+1=p-1$, as required.

A matching $M$ in a graph $G$ is a subset of edges no two of which have a common vertex. The maximum number of edges in a matching of a graph $G$ is called the matching number of $G$ and denoted $\beta(G)$. A matching is perfect if every vertex in $G$ is incident to some edge in the matching [10].

Theorem 2.8. Let $S_{M}=\left\{0, x, x^{2}, \ldots, x^{p}\right\}$ be a monogenic semigroups. Then the matching number of $G=\Gamma\left(S_{M}\right)$ is given by $\beta(G)=p-\left\lceil\frac{p}{2}\right\rceil$.

Proof. By the definition of $\Gamma\left(S_{M}\right)$, we get $x^{k} x^{p+1-k} \in E\left(\Gamma\left(S_{M}\right)\right)$ for $\left\lceil\frac{p}{2}\right\rceil+1 \leq k \leq p$. Then a maximum matching in $G$ is $M=\left\{x^{p} x, x^{p-1} x^{2}, \ldots, x^{\left\lceil\frac{p}{2}\right\rceil+1} x^{p-\left\lceil\frac{p}{2}\right\rceil}\right\}$. Finally, we get $\beta(G)=p-\left\lceil\frac{p}{2}\right\rceil$.

Remark 2.9. If $p$ is even, the graph $\Gamma\left(S_{M}\right)$ is the graph with perfect matchings.

Theorem 2.10. Let $S_{M}^{1}=\left\{0, x, x^{2}, \ldots, x^{p}\right\}$ and $S_{M}^{2}=\left\{0, y, y^{2}, \ldots, y^{s}\right\}$ be two monogenic semigroups with $p \geq s$. Then the matching number of $G=\Gamma_{1}+\Gamma_{2}$ is given by

$$
\beta(G)=\left\{\begin{array}{c}
\frac{p+s}{2}, \text { both } p \text { and } q \text { are odd or even } \\
\frac{p+s-1}{2}, \text { otherwise }
\end{array}\right.
$$

Proof. Let $S_{M}^{1}=\left\{0, x, x^{2}, \ldots x^{p}\right\}$ and $S_{M}^{2}=\left\{0, y, y^{2}, \ldots, y^{s}\right\}$ be two monogenic semigroups with $p \geq s$.

Case 1. Let $p$ and $s$ be even. In this case, by the definition of join product and theorem 2.8 we get $x^{k} x^{p+1-k} \in$ $E\left(\Gamma_{1}\right),\left\lceil\frac{p}{2}\right\rceil+1 \leq k \leq p$ and $y^{k} y^{s+1-k} \in E\left(\Gamma_{2}\right),\left\lceil\frac{s}{2}\right\rceil+1 \leq k \leq s$. Then a maximum matching in $G$ is $M=$ $\left.\left\{x^{p} x, x^{p-1} x^{2}, \ldots, x^{\left\lceil\frac{p}{2}\right\rceil+1} x^{p-\left\lceil\frac{p}{2}\right\rceil}, y^{s} y, y^{s-1} y^{2}, \ldots, y^{\left\lceil\frac{s}{2}\right\rceil+1} y^{s-\left\lceil\frac{p}{2}\right\rceil}\right\rceil\right)$. Finally, we get $\beta(G)=p+s-\left\lceil\frac{p}{2}\right\rceil-\left\lceil\frac{s}{2}\right\rceil=p+s-p / 2-s / 2=$ $(p+s) / 2$.

Case 2. Let $p$ and $s$ be odd. By similar argument, we get

$$
\left.M=\left\{x^{p} x, x^{p-1} x^{2}, \ldots, x^{\left\lceil\frac{p}{2}\right\rceil+1} x^{p-\left\lceil\frac{p}{2}\right\rceil}, y^{s} y, y^{s-1}\right) y^{2}, \ldots, y^{\left\lceil\frac{s}{2}\right\rceil+1} y^{s-\left\lceil\frac{s}{2}\right\rceil}, x^{\frac{p+1}{2}} y^{\frac{s+1}{2}}\right\} .
$$

Then we have

$$
\beta(G)=p+s-\left\lceil\frac{p}{2}\right\rceil-\left\lceil\frac{s}{2}\right\rceil+1=p+s-(p+1) / 2-(s+1) / 2+1=(p+s) / 2 .
$$


Case 3. Let $p$ and $s$ be even and odd, respectively. Then a maximum matching in $G$ is $M=\left\{x^{p} x, x^{p-1} x^{2}, \ldots, x^{\left\lceil\frac{p}{2}\right\rceil+1} x^{p-\left\lceil\frac{p}{2}\right\rceil}, y^{s} y, y^{s-1} y^{2}, \ldots, y^{\left\lceil\frac{s}{2}\right\rceil+1} y^{s-\left\lceil\frac{s}{2}\right\rceil}\right\}$. Finally , we get $\beta(G)=p+s-\left\lceil\frac{p}{2}\right\rceil-\left\lceil\frac{s}{2}\right\rceil=p+s-$ $\frac{p}{2}-\frac{s+1}{2}=\frac{p+s-1}{2}$. Proof is completed.

A subset $A$ of the vertex set $V(G)$ of a graph is collect the domination set if every vertex $V(G) \backslash A$ is joined to at least one vertex of $A$ by an edge. The domination number $\gamma(G)$ is the number of vertices in the smallest dominating set for $G[10]$.

Theorem 2.11. Let $S_{M}^{1}=\left\{0, x, x^{2}, \ldots, x^{p}\right\}$ and $S_{M}^{2}=\left\{0, y, y^{2}, \ldots, y^{s}\right\}$ be two monogenic semigroups. Then

$$
\gamma\left(\Gamma_{1}+\Gamma_{2}\right)=1 .
$$

Proof. By the definition of join operation, the vertex $x^{p}$ is adjacent to all the other vertices. That is, $x^{p} \sim x^{i}$ and $x^{p} \sim y^{j}$ for $i=1,2, \ldots, p-1$ and $j=1,2, \ldots, s$. So the dominating set consist of only the element $x^{p}$. Then the domination number of the graph $\Gamma_{1}+\Gamma_{1}$ is 1 .

The chromatic number of a graph $G$ is the smallest number of colors needed to color the vertices of $G$ so that no two adjacent vertices share the same color. The chromatic number of a graph $\mathrm{G}$ is denoted $\chi(G)$ [11].

Theorem 2.12 ( [7]). The chromatic number of $\Gamma\left(S_{M}\right)$ is equal to $\chi\left(\Gamma\left(S_{M}\right)\right)=1+\left\lceil\frac{p-1}{2}\right\rceil$.

We now give chromatic number of $\Gamma\left(S_{M}^{1}\right)+\Gamma\left(S_{M}^{2}\right)$.

Theorem 2.13. Let $S_{M}^{1}=\left\{0, x, x^{2}, \ldots, x^{p}\right\}$ and $S_{M}^{2}=\left\{0, y, y^{2}, \ldots, y^{s}\right\}$ be two monogenic semigroups. Then

$$
\chi\left(\Gamma_{1}+\Gamma_{2}\right)=2+\left\lceil\frac{p-1}{2}\right\rceil+\left\lceil\frac{s-1}{2}\right\rceil .
$$

Proof. By Theorem 2.12, we have $1+\left\lceil\frac{p-1}{2}\right]$ of chromatic number of the graph $\Gamma_{1}$. Also we have $1+\left\lceil\frac{s-1}{2}\right\rceil$ of chromatic number of the graph $\Gamma_{2}$. Since all vertices in the graph $\Gamma_{1}$ are adjacent all vertices of the graph $\Gamma_{2}$, we cannot use the colors we use in $\Gamma_{1}$ to color the vertices of the graph $\Gamma_{2}$. Then we get

$$
\chi\left(\Gamma_{1}+\Gamma_{2}\right)=2+\left\lceil\frac{p-1}{2}\right\rceil+\left\lceil\frac{s-1}{2}\right\rceil .
$$

Proof is completed.

A clique of a graph $G$ is a complete subgraph of $G$. The clique number of a graph $G$, denoted $\omega(G)$, is the maximum number of vertices in a complete subgraph of $G$.

Theorem 2.14. Let $S_{M}^{1}=\left\{0, x, x^{2}, \ldots, x^{p}\right\}$ and $S_{M}^{2}=\left\{0, y, y^{2}, \ldots, y^{s}\right\}$ be two monogenic semigroups. Then

$$
\omega\left(\Gamma_{1}+\Gamma_{2}\right)=2+\left\lceil\frac{p-1}{2}\right\rceil+\left\lceil\frac{s-1}{2}\right\rceil .
$$

Proof. Let $A=\left\{x^{\left\lceil\frac{p}{2}\right\rceil}, x^{\left[\frac{p}{2}\right\rceil+1}, \ldots, x^{p}\right\}$ be subset of the set $V\left(\Gamma_{1}\right)$ and $B=\left\{y^{\left\lceil\frac{s}{2}\right\rceil}, y^{\left\lceil\frac{p}{2}\right\rceil+1}, \ldots, y^{s}\right\}$ be subset of the set $V\left(\Gamma_{2}\right)$. Furthermore, all of the vertices in sets $A$ and $B$ are adjacent to each other. Then we get

$$
\omega\left(\Gamma_{1}+\Gamma_{2}\right)=|A|+|B|=\left(p-\left\lceil\frac{p}{2}\right\rceil+1\right)+\left(s-\left\lceil\frac{s}{2}\right\rceil+1\right) .
$$

Finally, the fact that $p-\left\lceil\frac{p}{2}\right\rceil=\left\lceil\frac{p-1}{2}\right\rceil$ and $q-\left\lceil\frac{q}{2}\right\rceil=\left\lceil\frac{q-1}{2}\right\rceil$, we get $\omega\left(\Gamma_{1}+\Gamma_{2}\right)=2+\left\lceil\frac{p-1}{2}\right\rceil+\left\lceil\frac{s-1}{2}\right\rceil$. So proof is completed. 


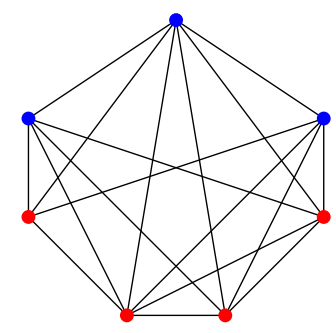

Figure 1: The graph $\Gamma_{1}+\Gamma_{2}$

Example 2.15. Let us consider the semigroups $S_{M}^{1}=\left\{x, x^{2}, x^{3}, x^{4}\right\}$ and $S_{M}^{2}=\left\{y, y^{2}, y^{3}\right\}$. Then the graph $\Gamma_{1}+\Gamma_{2}$ is given as drawn in Figure 1. Then we have
1. $\operatorname{diam}\left(\Gamma_{1}+\Gamma_{2}\right)=2 \quad$ (by Theorem 2.1.
2. $\operatorname{rad}\left(\Gamma_{1}+\Gamma_{2}\right)=1 \quad($ by Theorem 2.2).
3. $\operatorname{girth}\left(\Gamma_{1}+\Gamma_{2}\right)=3 \quad$ (by Theorem 2.3).
4. $\Delta\left(\Gamma_{1}+\Gamma_{2}\right)=6 \quad$ (by Theorem 2.4).
5. $\delta\left(\Gamma_{1}+\Gamma_{2}\right)=4 \quad($ by Theorem 2.5$)$.
6. $D S\left(\Gamma_{1}+\Gamma_{2}\right)=\{4,5,5,5,5,6,6\} \quad$ (by Theorem 2.7).
7. $t\left(\Gamma_{1}+\Gamma_{2}\right)=3 \quad$ (by Theorem 2.7).
8. $\beta\left(\Gamma_{1}+\Gamma_{2}\right)=3 \quad$ (by Theorem 2.10).
9. $\gamma\left(\Gamma_{1}+\Gamma_{2}\right)=1 \quad$ (by Theorem 2.11).
10. $\chi\left(\Gamma_{1}+\Gamma_{2}\right)=5 \quad$ (by Theorem 2.13).
11. $\omega\left(\Gamma_{1}+\Gamma_{2}\right)=5 \quad$ (by Theorem 2.14).

\section{CONFLICTS OF INTEREST}

The author declares that there are no conflicts of interest regarding the publication of this article.

\section{REFERENCES}

[1] Akgunes, N., Das, K.C., Cevik, A.S., Cangul, I.N., Some properties on the lexicographic product of graphs obtained by monogenic semigroups, J. Inequal. Appl., 238(2013), 1-9.

[2] Akgunes, N., Das, K. Ch., Cevik, A.S., Some properties on the tensor product of graphs obtained by monogenic semigroups, Appl. Math. Comput., 235(2014), 352-357.

[3] Anderson, D.F., Livingston, P.S., The zero-divisor graph of commutative ring, Journal of Algebra, 217(1999), 434-447.

[4] Anderson, D.F., Badawi, A., On the zero-divisor graph of a ring, Comm. Algebra, 36(2008), 3073-3092.

[5] Anderson, D.D., Naseer, M., Beck's coloring of a commutative ring, Journal of Algebra, 159(1991), 500-514.

[6] Beck, I., Coloring of commutating ring, Journal of Algebra, 116(1988), 208-226.

[7] Das, K.C., Akgunes, N., Cevik, A.S., On a graph of monogenic semigroup, Journal of Ineq.and Appl., 44(2013), 1-13.

[8] DeMeyer, F.R., McKenzie, T., Schneider, K., The zero-divisor graph of a commutative semigroup, Semigroup Forum, 65(2002), $206-214$.

[9] DeMeyer, F.R., DeMeyer, L., Zero-divisor graphs of semigroups, Journal of Algebra, 283(2005), 190-198.

[10] Gross, J.L., Yellen, J., Handbook of graph theory, Chapman Hall, CRC Press 2004.

[11] Klavžar, S., Coloring graph products - a survey, Discrete Math., 155(1996), 135-145.

[12] Mukwembi, S., A note on diameter and the degree sequence of a graph, Appl. Math. Lett., 25(2002), 175-178. 
[13] Nacaroglu, Y., On the corona product of monogenic semigroup graphs, Adv. and Appl. in Discrete Math., 19(2018), 409-420.

[14] Nacaroglu, Y., Akgunes, N., On the sigma index of the corona products of monogenic semigroup graphs, JUM., 2 (1)(2019), 68-74. 